\title{
Energieeffiziente Planung von Wertschöpfungsnetzwerken
}

\author{
Integration von Energieeffizienz in die strategische Gestaltung \\ von Produktions- und Logistiknetzwerken
}

\author{
Lucas Schreiber, Lea Vliegen, Jan-Philipp Jarmer, Andreas Günter und Christian Hohaus, Fraunhofer \\ IML, Dortmund, David Grimm, LogProlT GmbH, Dortmund, Andrea Vennemann, thyssenkrupp Materi- \\ als Services und Christian Fischer, Behr-Hella Thermocontrol GmbH
}

\begin{abstract}
Bei der Auswahl eines neuen Kühlschranks ist die Energieeffizienz heutzutage ein selbstverständliches Auswahlkriterium. In der strategischen und taktischen Planung von Wertschöpfungsnetzwerken ist dies noch nicht der Fall. Mit der im Forschungsprojekt $E^{2}$-Design entwickelten Toolbox wird eine Berücksichtigung von Energieeffizienz neben den klassischen Leistungs- und Kostengrößen frühzeitig im Planungsprozess von Produktions- und Logistiknetzwerken ermöglicht. Im vorliegenden Beitrag werden die zugrundeliegenden Energiedaten und die entwickelten Planungswerkzeuge vorgestellt sowie der Nutzen aus Anwenderperspektive abgeleitet. Auf Basis des vorgestellten methodisch unterstützten Ansatzes ist es möglich, die grundlegenden Supply Chain Design (SCD)-Entscheidungen frühzeitig unter Berücksichtigung der Energieeffizienz zu treffen, um aus dem Gesamtpotenzial zu schöpfen.
\end{abstract}

Bei der Betrachtung des Endenergieverbrauchs in Deutschland im Jahr 2018 wird deutlich, dass die Bereiche Industrie (Produktion) und Verkehr (Logistik) zusammen einen Anteil von $59,6 \%$ ausmachen [1]. Um Wertschöpfungsnetzwerke wirtschaftlich und energieeffizient zu konfigurieren, ist es erforderlich diese Zielgrößen bereits in der strategischen und taktischen Planung zu berücksichtigen. Die Planung im SCD ist in einzelne Aufgaben wie die Standortwahl, Partnerauswahl oder Kapazitätsdimensionierung zu unterteilen [2]. Für die verschiedenen Aufgaben sind Ansätze zu entwickeln, mithilfe derer die meist operative Kennzahl Energieeffizienz für die strategische Planung zu abstrahieren ist, damit sie frühzeitig in der strategischen Planung berücksichtigt werden kann.

Auf Basis von Energiedaten sowie weiteren Unternehmensdaten wurden für die zentralen SCD-Aufgabenstellungen unterstützende, zukunftsgerichtete Werkzeuge erstellt. Um unternehmensspezifische Energieverbräuche auf Standort- sowie Netzwerkebene bei der Planung zu erfassen und zu berücksichtigen, sind zusätzliche Messungen von Stromverbräuchen durchzuführen.

Abhängig von der Gewichtung der Zielgrößen für das Wertschöpfungsnetzwerk, wie
Wirtschaftlichkeit oder Energieeffizienz, werden assistiert durch das Werkzeug der Simulation potentielle zukünftige Netzwerkgestaltungen bewertet. Durch iterative Schleifen ist es möglich, vielversprechende Parametrierungen für das gewünschte, zukünftige Netzwerk zu bestimmen.

\section{Generierung einer Energiedaten- basis}

Ziel der Energiedatenbank ist, relevanten Kostenblöcken im SCD äquivalente Energieverbräuche zuzuordnen [3]. Energieverbräuche können zum einen individuell gemessen und zum anderen aus Datenbanken mit entsprechenden Prozessen oder Produkten in Verbindung gebracht werden. Eine gängige Methode zur Darstellung von Energieverbräuchen ist der "Kumulierte Energieaufwand" (KEA) [4]. Der KEA beschreibt die Summe des primären Energieinputs und wird in drei Phasen unterteilt: Produktion, Nutzung und Entsorgung. Die Phasen der Nutzung und der Entsorgung sind in der Regel nicht Bestandteil des SCD, sodass der Fokus der Datenbeschaffung auf die dazugehörigen Transformations- und Distributionsprozesse in der Phase der Produktion gelegt wird. Dazu zählen die Beschaffung von für die Produktion notwendigen Rohstoffen, die Ver-
Lucas Schreiber, M. Sc. arbeitet als wissenschaftlicher Mitarbeiter in der Abteilung Supply Chain Engineering am Fraunhofer-Institut für Materialfluss und Logistik IML in Dortmund.

Lea Vliegen, M. Sc. arbeitet als wissenschaftliche Mitarbeiterin in der Abteilung Produktionslogistik am Abtellung Produktionslogistik am und Logistik IML in Dortmund.

Jan-Philipp Jarmer, M. Sc. arbeitet als wissenschaftlicher Mitarbeiter in der Abteilung Umwelt und Ressourcenlogistik am Fraunhofer-Institut cenlogistik am Fraunhofer-Institut
für Materialfluss und Logistik IML in Dortmund.

Andreas Günter, M. Sc. arbeitet als wissenschaftlicher Mitarbeiter in der Abteilung Produktionslogistik am Fraunhofer-Institut für Materialfluss und Logistik IML in Dortmund.

Christian Hohaus, Dipl.-Ing. arbeitet als wissenschaftlicher Mitarbeiter in der Abteilung Umwelt und Ressourcenlogistik am Fraunhofer-Institut für Materialfluss und Logistik IML in Dortmund.

David Grimm, M. Sc. ist Mitarbeiter be der LogProlT GmbH.

Andrea Vennemann, M. Sc. arbeitet als Projektmanagerin in der Abteilung Supply Chain Management bei der thyssenkrupp Schulte $\mathrm{GmbH}$.

Christian Fischer arbeitet in der internationalen Logistikplanung bei der Behr-Hella Thermocontrol GmbH.

e2design@iml.fraunhofer.de www.iml.fraunhofer.de 


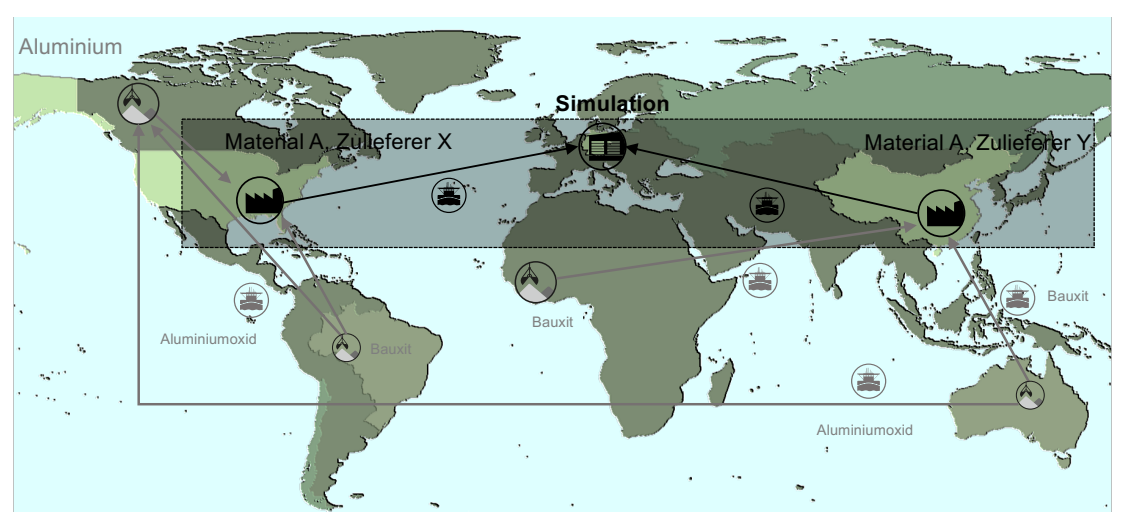

Bild 1: Exemplarische Darstellung globaler Transformations- und Distributionsprozesse.

arbeitung bzw. Produktion von (Zwischen-)Produkten sowie Transporte. Die Verfügbarkeit und Nutzung von Daten zur Berechnung des KEA in Wertschöpfungsnetzwerken in den genannten Prozessen wird im Folgenden näher betrachtet.

\section{Beschaffung}

Bei der energetischen Bewertung von Beschaffungsbeziehungen sind Unternehmen mit komplexen Wertschöpfungsnetzwerken und unvollständigen Informationen konfrontiert. Mithilfe von Informationen über den Standort des Zulieferers sowie einer Materialliste unterstützt das Rohstoffmodell [5] dabei, eine erste energetische Indikation für beschaffte Materialien oder Produkte, inkl. des Abbaus bzw. der Herstellung der Vorproukte, abzuschätzen. Dazu werden aus Datenbanken, z. B. ecoinvent [6], regionenspezifische Datensätze mithilfe der KEA-Methode für verschiedene Verarbeitungsstufen ausgewertet und aggregiert. Bei Metallen werden in der Regel die Stufen Abbau, Schmelze und Raffination betrachtet. Da Zwischenprodukte dieser Verarbeitungsstufen global gehandelt werden, integriert das Rohstoffmodell neben den energetischen Aufwendungen für die Transformationsprozesse auch die energetischen Aufwendungen für die Distributionsprozesse. Die Handelsrelationen werden aus der UN-Datenbank Comtrade abgeleitet. Als Ergebnis gibt das Rohstoffmodell einen Energiewert pro Material pro Land aus, der verschiedene Verarbeitungsstufen und globale Handelsbeziehungen berücksichtigt und in weitere unternehmensinterne Assistenzsysteme zur Entscheidungsfindung (z. B. Simulation) eingebunden werden kann.

\section{Produktion}

Für belastbare energetische SCD-Entscheidungen auf der Ebene des Produktionsstandorts, ist es notwendig, dass neben dem Gesamtverbrauch eines Standorts wichtige Funktionsbereiche mit entsprechender Ausstattung bestimmt und charakterisiert werden. Eine Möglichkeit der Charakterisierung ist dabei die Unterscheidung von Energieverbräuchen in Grundenergieverbrauch, variabler Energieverbrauch und sonstiger Zusatzverbrauch.

Nach erfolgreicher energetischer Charakterisierung der Funktionsbereiche mit entsprechender Ausstattung erfolgt die Allokation der Energieverbräuche mit dem Produktionsprogramm. So ist es möglich, Energieverbräuche in Abhängigkeit der zu erwartenden Aufträge zu prognostizieren oder Reallokationen von Produkten innerhalb eines Standorts oder innerhalb des Produktionsnetzwerks zu initiieren und im Rahmen von strategischen Entscheidungen Kennzahlen wie Energieverbrauch pro Produkt oder Energieverbrauch pro Periode pro Funktionsbereich zu optimieren.

\section{Transport}

Für die Evaluation der Phase Transport liefert das Global Logistics Emission Council (GLEC) eine umfangreiche Beschreibung für die Berechnung von Emissionen bzw. Energieverbräuchen für verschiedenen Transportträger [7]. Für den Fall, dass der Zugriff auf Primärverbrauchsdaten nicht möglich ist, stellt das „Handbuch für Emissionsfaktoren des Straßenverkehrs (HBEFA)" entsprechende Durchschnittswerte zur energetischen Abschätzung je gefahrenem Tonnenkilometer zur Verfügung [8].

Mithilfe dieser Bausteine lässt sich eine Energiedatenbank aufbauen, die ein SCD aus der energetischen Perspektive erlaubt. Zwar ist die regionale Auflösung der Datensätze mit den dazugehörigen Prozessen zur Herstellung der Vorprodukte bislang in der Regel auf wenige Wirtschaftsräume bzw. Kontinente beschränkt, die kontinuierliche Forschung in diesem Themenfeld lässt jedoch eine Verbesserung der Auflösung der Daten erwarten.

\section{$E^{2}$-Design-Planungstoolbox}

Um die konzeptionierte und implementierte Energiedatenbank neben der Informationsgenerierung auch analytisch nutzbar zu machen, wurden mehrere methodische Werkzeuge zur strategischen Konfiguration von Supply Chains entwickelt, welche direkt auf die Energiedatenbank zugreifen. Die resultierende Planungstoolbox basiert auf einem mehrstufigen Vorgehensmodell für die integrierte, energieeffizienzorientierte (Um-) Gestaltung von Produktions- und Logistiknetzwerken unter Berücksichtigung energetischer Zielgrößen [9]. Analog zur Energiedatenbank werden die Di- 


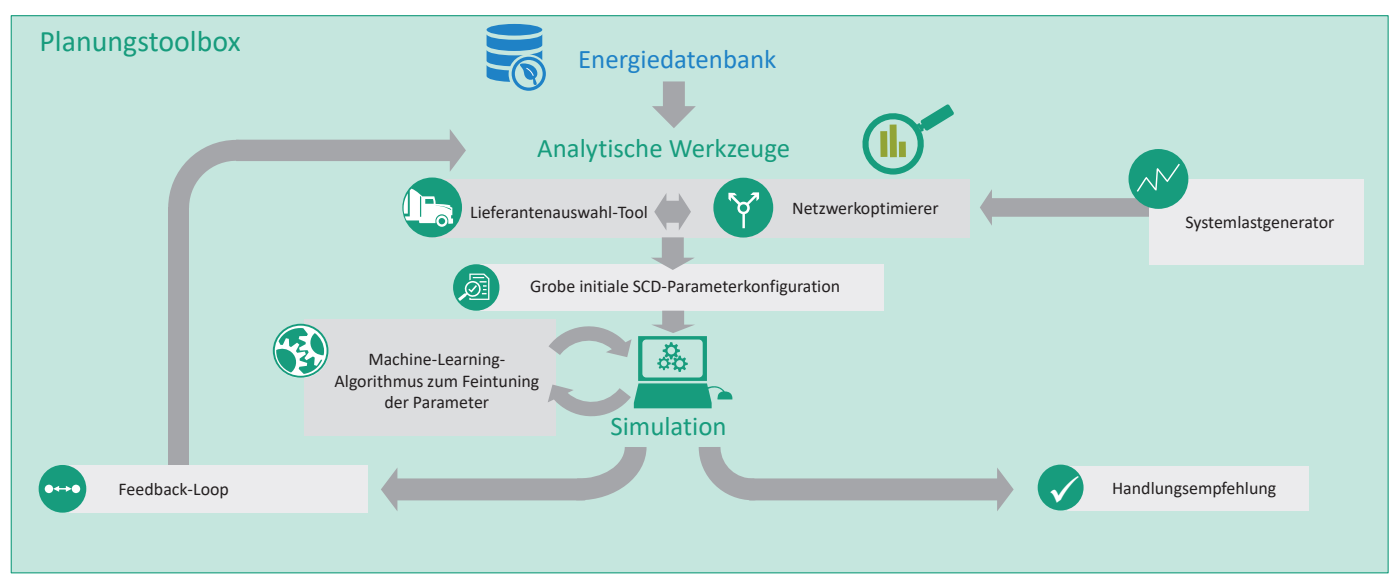

mensionen Beschaffung, Produktion und Transport adressiert. Die Nutzung von variierenden Absatzprognosen als Systemlast befähigt zudem zur Analyse von mehreren strategischen Szenarien. Eine Übersicht der Zusammenhänge und Bestandteile der Planungstoolbox kann dem Bild 2 entnommen werden.

Für die Generierung eines Szenarios ist neben der Anbindung der spezifischen Energiedaten sowie der Gewichtung des Zielsystems auch die zukünftig erwartete Nachfragesituation als Planungsgrundlage zu integrieren. Zur Adressierung dieser initialen Anforderung wurde ein Auftragsgenerator konzeptioniert und entwickelt, welcher zum einen die unternehmensspezifischen Vergangenheitsdaten (bspw. Absatzhistorie) und zum anderen erwartete Veränderungen von zentralen Eckwerten (bspw. Absatzentwicklung einzelner Märkte oder Produktgruppen) berücksichtigt.

Als analytische Werkzeuge dienen ein Lieferantenauswahltool sowie ein Netzwerkoptimierer, durch welche die Belegung der Knoten und Kanten des Netzwerks initial konfiguriert werden können. Mithilfe der Fuzzy-Logik (angelehnt an [10]) und linearen Optimierungsmodellen (angelehnt an [11]) können basierend auf individuellen Kriteriengewichtungen grobe initiale SCD-Parameterkonfigurationen generiert werden. Dazu zählen die Auswahl von Lieferanten, Standorten und Distributionskanälen sowie die Festlegung von benötigten Kapazitäten und Transportmedien.

Diese statisch erzeugte grobe initiale Parameterkonfiguration ist im dynamischen Wirkungszusammenhang zu bewerten, da eine statische Konfiguration keine exakten Aussagen über die Performance im zeitlichen Ablauf des Zusammenspiels von Beschaffung, Produktion und Distribution treffen kann. Für die Bewertung und Feinplanung wird auf das Werkzeug der er- eignisdiskreten Simulation zurückgegriffen. Mithilfe der Ankopplung eines Machine Learning Algorithmus [12] nach VDI Richtlinie 3663 Blatt 12 Kategorie D [13] werden Eingangsparameter wie der Meldebestand, Richtbestand als auch die Materialallokation pro Produkt und Standort variiert. Der eingebundene Algorithmus lernt mit jedem neuen Simulationslauf defizitäre Lösungsparameter zu meiden und den Untersuchungsraum in Richtung vielversprechender Parameter-Sets genauer zu betrachten.

Die Auswertung der Kennzahlen kann einerseits aufzeigen, dass die dynamische Nachsteuerung die Ergebnisse der statischen strategischen Gestaltung verzerrt hat oder die Ergebnisse basierend auf der initialen Gewichtung nicht zufriedenstellend sind. Für diesen Fall ist eine Feedback-Schleife zu durchlaufen und die Kriteriengewichtung der ersten Planungsschritte anzupassen. Sollten die Ergebnisse zufriedenstellend sein, können Handlungsempfehlungen für das reale Wertschöpfungsnetzwerk abgeleitet werden.

Um die Planungstoolbox für eine breite Masse zur Verfügung zu stellen, sind die entwickelten Module über eine cloudbasierte Plattform nutzbar gemacht worden. Auf der mit dem Umsetzungspartner LogProlT aufgebauten Microsoft Azure Cloud können die Module anwendungsfallspezifisch kombiniert werden, um individuelle Gegebenheiten unterschiedlicher Supply Chains zu berücksichtigen (Bild 3). Ein Szenario-Manager ermöglicht die parallele Erstellung mehrerer Szenarien, um Trade-Off-Effekte zwischen Kosten, Performance und Energieeffizienz unmittelbar vergleichbar zu machen.

\section{Validierung und Nutzen aus der Anwenderperspektive}

Zur Validierung der $E^{2}$-Design-Planungstoolbox unterstützen die Anwendungspartner
Bild 2: Bestandteile und Zusammenhänge der $E^{2}$-Design-Planungstoolbox.

1] BMWi: Energieeffizienz in Zahlen. URL: www.bmwi de/Redaktion/DE/Publikationen/Energie/energieeffizienz-in-zahlen-2020.pdf? blob=publicationFile $\& v=\overline{2} 0$ Abrufdatum 24.02.2021.

[2] Parlings, M.; Cirullies, J.; Klingebiel, K.: A literature-based state of the art review on the dentification and classification of supply chain design tasks. In: Disruptive supply network models in future industrial systems, configuring for resilience and sustainability. Cambridge, UK 2013

[3] Schreiber, L.; Jarmer, J.P.; Kamphues, J.: Energy-efficient Supply Chain Design: Data Aggregation and Processing In: Proceedings of the Hamburg International Conference of Logistics (HICL). Hamburg 2020.

[4] VDI 4600: Kumulierter Energieaufwand (KEA) - Begriffe, Berechnungsmethoden. In: VDI-Gesellschaft Energie und Umwelt (Hrsg): Fachbereich Integrale Energie- und Umweltfragen. Düsseldorf 2012.

[5] Jarmer, J. P.; Hohaus, C.; Gronau, P.: Procedure model for integrating energy efficiency in strategic sourcing of electronic parts and components in the automotive sector - A case study. In: Conference Proceedings "Electronics Goes Green 2020". Berlin 2020

[6] Wernet, G.; Bauer, C.; Steubing, B.; Reinhard, J.; Moreno-Ruiz, E.; Weidema, B.: The ecoinvent database version 3 (part I): overview and methodology. In: Int J Life Cycle Assess 21 (2016) 9, S. 1218-1230.

[7] Smart Freight Centre: Global Logistics Emissions Council Framework for Logistics Emissions Accounting and Reporting. Version 2.0. Amsterdam 2019.

[8] Notter, B.; Keller, M.; Althaus, H. J.; Cos, B.; Knörr, W.; Heidt C. u. a.: HBEFA 4.1 - Development Report. Handbook of Emission Factors for Road Transport. Bern 2019.

[9] Schreiber, L.: Optimization and simulation for sustainable supply chain design. In: Proceedings of the Hamburg International Conference of Logistics (HICL). Hamburg 2019. 


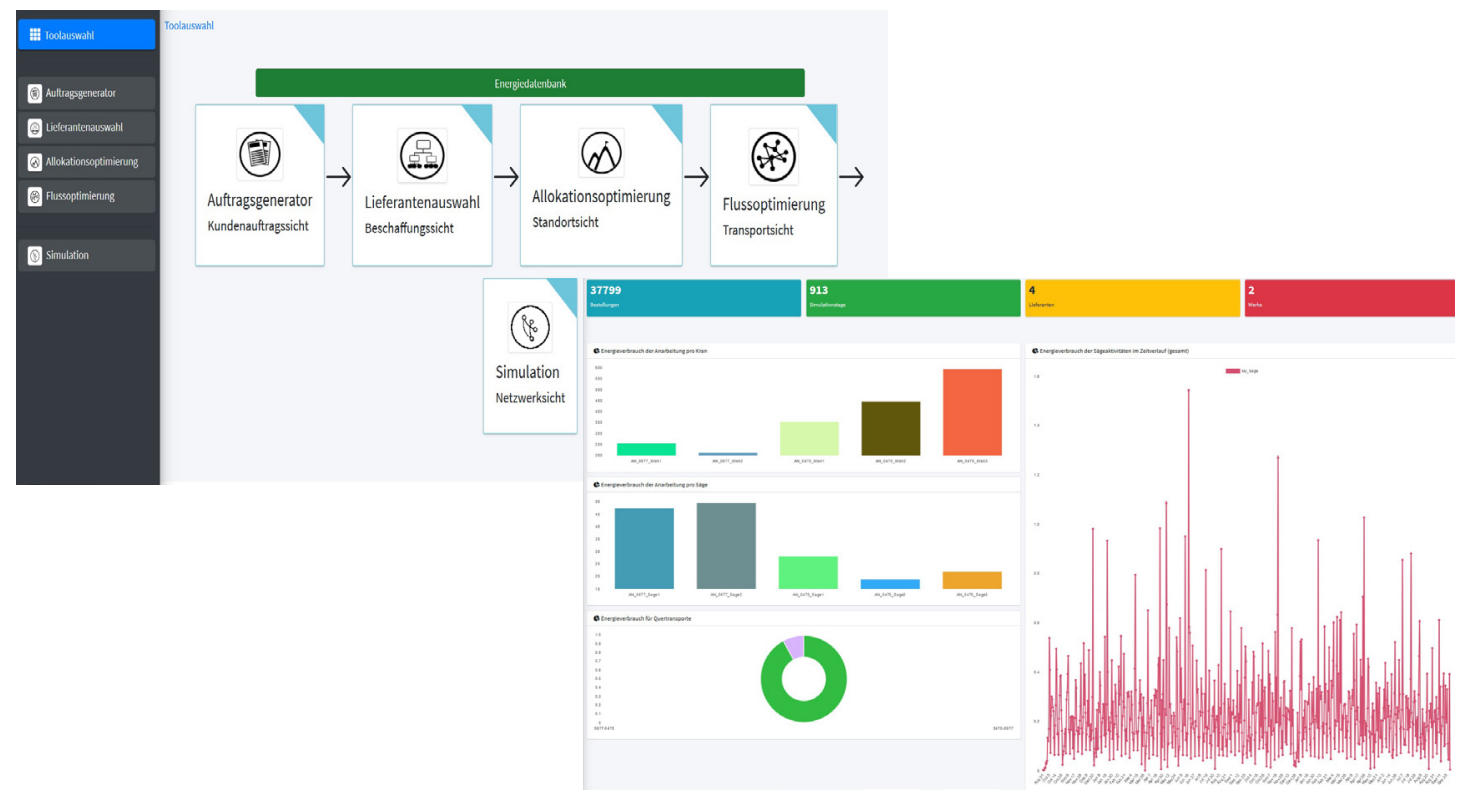

zung in den Modulen aufgebaut werden. Die Integrationsfähigkeit der Module wurde von thyssenkrupp Materials Services besonders hervorgehoben [14]. Für die nachhaltige Umgestaltung der Netzwerkstrukturen ist die Integration einer ökonomischen und ökologischen Betrachtungsweise zwingend erforderlich. Sie sind Kernaspekte für zukunftsträchtige Netzwerkgestaltungen und werden in den Unternehmensstrategien der Anwendungspartner

Bild 3: Auszug der Moduleinbindung auf die cloudbasierte Plattform.
[10] Csutora, R.; Buckley, J. J.: Fuzzy hierarchical analysis: The Lambda-Max method. In: Fuzzy Sets and Systems 120 (2001) 2, S. 181-195.

[11]Wang, F.; Lai, X.; Shi, N.: A multi-objective optimization for green supply chain network design. In: Decision Support Systems 51 (2010) 2, S. 262-269.

[12] Deb, K.; Pratap, A.; Agarwal, S.; Meyarivan, T: A fast and elitist multiobjective genetic algorithm: NSGA-II. In: IEEE Transactions on Evolutionary Computation 6 (2002) 2, S. 182-197.

[13] VDI 3633 Blatt 12: Simulation von Logistik-, Materialflussund Produktionssystemen Simulation und Optimierung. In: VDI-Gesellschaft Produktion und Logistik (Hrsg): VDI-Fachbereich Fabrikplanung und -betrieb. Düsseldorf 2020.

[14] Daniel Fruhner, D.; Grimm, D.; Sardesai, S.; Wagenitz, A. Vennemann, A.; Hegmanns, T.: A Tool-Independent Generalized Description for Sus tainable Supply Chain Design. In Proceedings of the 19th International Conference on Modeling \& Applied Simulation (MAS 2020). 2020
Behr-Hella Thermocontrol und thyssenkrupp Materials Services das Forschungsvorhaben. Mithilfe der Anwendungsfälle konnten industrierelevante Fragestellungen identifiziert werden und der ganzheitliche Planungsansatz anhand von Daten und Problemstellungen aus der industriellen Praxis validiert werden. Gemeinsam mit Behr-Hella Thermocontrol wurden verstärkt die Beschaffungsprozesse in der Automobilindustrie betrachtet. Aufgrund der Globalisierung, muss für eine Verbesserung der Energiebilanz die gesamte Wertschöpfungskette entlang der Supply Chain berücksichtigt werden. Aus der Anwendersicht ist das Projekt insbesondere im Rahmen der n-tier-Transparenz im Lieferantennetzwerk interessant. Als besondere Herausforderung ist hier die Transparenz in der Beurteilung der Energiebilanz von Rohstoffen und Zukaufteilen zu sehen, die aus einem Lieferantennetzwerk bezogen werden, da sie bei der Gesamtenergiebilanz berücksichtigt werden müssen [3]. Aus diesem Grund sind die Gestaltung der Beschaffungsprozesse, die Auswahl der Lieferanten und die Gestaltung der Transportrelationen als entscheidende Faktoren bei der nachhaltigen Optimierung des ökologischen Fußabdrucks zu berücksichtigen.

Im zweiten Anwendungsfall erlangte thyssenkrupp Materials Services Transparenz hinsichtlich der Energieverbräuche an den einzelnen Standorten inkl. einzelner Funktionsbereiche im Produktionsnetzwerk. Insbesondere im Hinblick auf die Produktion und Distribution konnte durch spezifische Energiemessungen an ausgewählten Standorten, angereichert durch Kosten- und Leistungsparameter, eine industriell relevante Datengrundlage zur Nut- bereits fest verankert. Das im Projekt $E^{2}$-Design entwickelte Planungsinstrumentarium besteht aus ineinandergreifenden Planungsmodulen, die eine fundierte Grundlage zur langfristigen Beurteilung von dynamischen Effekten in der Logistik ermöglicht. Dies ist insbesondere vor dem Hintergrund des zunehmenden digitalen und schnelllebigen industriellen Umfelds von enormer Wichtigkeit.

\section{Zusammenfassung}

Die Anwenderperspektiven verdeutlichen, dass die $E^{2}$-Design-Planungstoolbox die methodischen Grundlagen notwendigen - jedoch weitestgehend nicht vorhandenen - Transparenz und Integrationsfähigkeit von energetischen Kenngrößen mithilfe praxistauglicher Werkzeuge befähigt. Durch eine frühe Berücksichtigung der Energieeffizienz im Planungsprozess mittels methodisch fundierter Werkzeuge sowie einer aussagekräftigen Energiedatenbank, sind die größten Energieeffizienzpotenziale zu heben, ohne dass eine signifikante Steigerung der Kosten oder ein Serviceverlust verzeichnet werden müssen.

Dieser Beitrag entstand im Rahmen des Projekts "E ${ }^{2}$-Design: Energieeffizienz in der strategischen Gestaltung von Produktions- und Logistiknetzwerken", das vom Bundesministerium für Wirtschaft und Energie unter dem Kennzeichen 03ET1558 A-D gefördert wird.

\section{Schlüsselwörter:}

Energieeffizienz, Supply Chain Design, Nachhaltigkeit, strategische Planung, Energiedatenbank, Optimierung, Simulation 